\title{
Omics of complex environmental microbial communities
}

\author{
SU DING ${ }^{1}$, NICOLE J. BALE ${ }^{2}$, ELLEN C. HOPMANS ${ }^{2}$, \\ LAURA VILLANUEVA ${ }^{2,3}$, MILOU G.I. ARTS ${ }^{2,3}$, STEFAN \\ SCHOUTEN $^{4,5}$ AND JAAP S. SINNINGHE DAMSTÉ 2,3 \\ ${ }^{1}$ Royal Netherlands Institute for Sea Research \\ ${ }^{2} \mathrm{NIOZ}$ Royal Netherlands Institute for Sea Research \\ ${ }^{3}$ Faculty of Geosciences, Utrecht University \\ ${ }^{4}$ NIOZ-Royal Netherlands Institute for Sea Research \\ ${ }^{5}$ Utrecht University \\ Presenting Author: su.ding@nioz.nl
}

Over recent decades, advances in analytical and sampling techniques have paved the way for significant improvements in understanding the field of organic biogeochemistry. One of the most recent of these advances in the impressive growth of 'omics' (e.g. genomics and lipidomics), which has ushered the field into a new era [1]. For instance, traditional detection of lipids based on individual peak identification resulted in a limited data acquisition of certain target lipid groups. Conversely nontargeted lipidomic approaches allow for comprehensive lipidome profiling without a priori mass spectrometry knowledge. In addition, integration of omics technologies may provide us a more complete picture of the composition and functions of lipids and their related microbial communities in the environment. Here, we explore a comprehensive profiling of microbial communities and their lipids throughout the water column of a marine euxinic basin (i.e. Black Sea) using 16S rRNA gene amplicon sequencing and ultra high-pressure liquid chromatography coupled with high-resolution tandem mass spectrometry (UHPLC-HRMS/MS). An information theory framework combined with molecular networking [2] based on the similarity of the mass spectra of lipids (Fig. 1A) enabled us to capture lipidomic diversity and specificity in the environment, identify novel lipids, differentiate microbial sources within a lipid group, and discover potential biomarkers for biogeochemical processes. For instance, 1-deoxyceramides and their derivatives with different polar moieties were tentatively identified by using the molecular network approach. The deep, euxinic waters $(500$ to $2000 \mathrm{~m})$ had the most specialized lipidome profiles among all the water column depth samples. Furthermore, lipidome profile and an abundance profile of $16 \mathrm{~S}$ diversity based on 16S rRNA gene amplicon sequencing data and total abundance values were used and a new tool called 'mmvec' was applied [3] to infer microbe-lipid connections through their co-occurrence probabilities (Fig. 1B and C). The workflow presented here allows to comprehensively recognize novel lipid biomarkers linked to uncultivated microbes as well as important biogeochemical processes in the environment.

\section{References}

[1] Steen, A. D. et al. (2020), Frontiers in Marine Science 7

[2] Nothias L.-F. et al. (2020), Nature Methods 17, 905-908

[3] Morton, J. T. et al. (2019), Nature Methods 16, 1306-1314

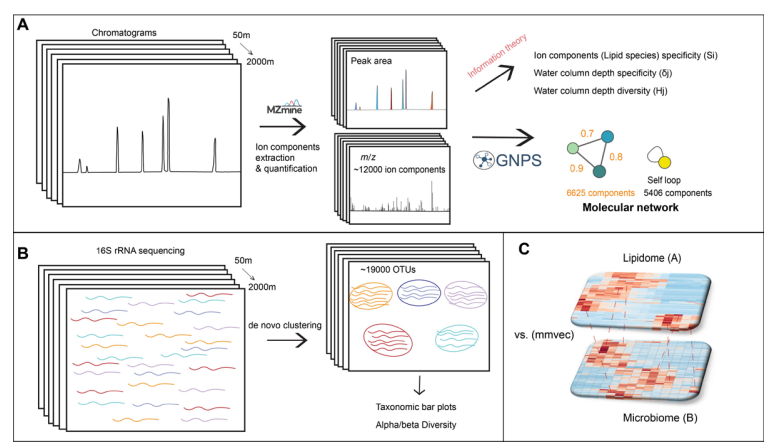

